\title{
APPROXIMATION NUMBERS AND KOLMOGOROFF DIAMETERS OF BOUNDED LINEAR OPERATORS
}

\author{
BY C. V. HUTTON ${ }^{1}$, J. S. MORRELL ${ }^{2}$ AND J. R. RETHERFORD ${ }^{3.4}$
}

Communicated by P. R. Halmos, September 22, 1973

0. Introduction. In this abstract we state some results concerning the approximation numbers and Kolmogoroff diameters of various operators between Banach spaces. Let $\mathscr{L}(E, F)$ and $\mathscr{F}(E, F)$ denote the bounded linear operators and the closure of the finite rank operators, respectively.

Following Pietsch [8], the $n$th approximation number $\alpha_{n}(T)$, of a $T \in \mathscr{L}(E, F)$ is defined as follows:

$$
\alpha_{n}(T)=\inf \{\|T-A\|: \operatorname{rank} A \leqq n\} ;
$$

the $n$th Kolmogoroff diameter of a $T \in \mathscr{L}(E, F)$ is defined by

$$
d_{n}(T)=\inf \left\{\left\|Q_{G} T\right\|: \operatorname{dim} G \leqq n\right\} .
$$

Here the infimum is over all subspaces $G \subset F$ and $Q_{G}$ denotes the canonical quotient map of $F \rightarrow F / G$.

It is clear that $\alpha_{n}(T)$ and $d_{n}(T)$ are monotone decreasing sequences and that $\lim _{n} \alpha_{n}(T)=0$ if and only if $T$ is the limit of finite rank operators and $\lim _{n} d_{n}(T)=0$ if and only if $T$ is compact.

For a brief discussion of the algebraic and analytic properties of $\alpha_{n}$ and $d_{n}$ (and other characteristics of bounded linear operators) see [7]. For compact operators on Hilbert space $\alpha_{n}(T)=d_{n}(T)$ and this characteristic has been extensively studied in the book of Gohberg and Krein [1]. For arbitrary Banach spaces the results are few. Some very interesting results can be found in the papers [5] and [6]; related results are also to be found in the classic memoir of Grothendieck, second part [2].

AMS (MOS) subject classifications (1970). Primary 47A30, 47B05, 47B10.

${ }_{1}$ Portions of this work appear in the dissertation of the first named author written at Louisiana State University under the direction of the third named author.

${ }^{2}$ Research supported in part by an NSF Science Development Grant to Louisiana State University and by a Faculty Research Council Grant from the University of Southern Mississippi.

${ }^{3}$ Portions of this paper were presented by the third author, as part of an invited address, at the NSF Regional Conference on Approximation Theory held at Kent State University, June 11-15, 1973.

${ }^{4}$ Research supported by NSF-GP-34193. 
Except where noted all our notation is standard. Details of the following results will appear elsewhere.

1. General relationships between $\alpha_{n}$ and $d_{n}$.

1.1. Theorem. For any $T \in \mathscr{L}(E, F)$ the following inequality is valid:

$$
d_{n}(T) \leqq \alpha_{n}(T) \leqq(\sqrt{ } n+1) d_{n}(T), \quad \text { for each } n .
$$

The best value, $p(n)$, for which $\alpha_{n}(T) \leqq p(n) d_{n}(T)$ is not known. In general, $p(n)$ cannot be replaced by a constant $K$.

If the domain space is nice enough, however, $\alpha_{n}$ and $d_{n}$ always coincide. More precisely,

1.2. THEOREM. If $E$ is an $L_{1}(\mu)$-space then for any $T \in \mathscr{L}(E, F)$, $F$ arbitrary, $\alpha_{n}(T)=d_{n}(T)$ for each $n$. In particular, if $E$ is a Banach space and $q$ a norm one surjection of $L_{1}(\mu)$ onto $E$, then for any $T \in \mathscr{L}(E, F)$, $d_{n}(T)=\alpha_{n}(T q)$ for each $n$.

2. Diagonal operators on the $l_{p}$-spaces. An operator $T$ of the form $T e_{n}=\lambda_{n} e_{n}$ where $\left(e_{n}\right)$ denotes the unit vectors in any $l_{p}$-space is called a diagonal operator and we say that $T$ corresponds to $\left(\lambda_{n}\right)$.

2.1. Theorem. Suppose $\left(\lambda_{n}\right)$ is an increasing sequence with limit $\lambda$. Then

(1) if $T: l_{1} \rightarrow l_{\infty}$ corresponds to $\left(\lambda_{n}\right), d_{n}(T)=\alpha_{n}(T)=\lambda / 2$ for all $n$; and, if $T: l_{1} \rightarrow c_{0}$ then $d_{n}(T)=\alpha_{n}(T)=\lambda$ for all $n$;

(2) if $T: l_{p} \rightarrow l_{\infty}, p>1, \alpha_{n}(T)=\lambda$ for all $n$ and $d_{n}(T)=\lambda / 2^{1 / p}$ for all $n$;

(3) if $1 \leqq p \leqq q<\infty$ and $T: l_{p} \rightarrow l_{q}$ then $d_{n}(T)=\alpha_{n}(T)=\lambda$ for all $n$.

If one considers the natural injection $I$ of $l_{1} \rightarrow c_{0}$, (1) above shows that $\alpha_{n}(I) \neq \alpha_{n}\left(I^{*}\right)$ and (2) above shows that, in general, $\alpha_{n}(T) \neq d_{n}(T)$.

2.2. THEOREM. Suppose $\left(\lambda_{n}\right)$ is a decreasing sequence.

(4) If $1 \leqq q<p<\infty$ and $T: l_{p} \rightarrow l_{q}$ corresponds to $\left(\lambda_{n}\right)$ then

where $1 / r+1 / p=1 / q$;

$$
d_{n}(T)=\alpha_{n}(T)=\left(\sum_{n+1}^{\infty}\left|\lambda_{i}\right|^{r}\right)^{\frac{1}{r}}
$$

(5) If $1 \leqq p<q<\infty$ then

$$
\left(\sum_{1}^{n+1} \lambda_{i}^{-r}\right) \frac{1}{r} \leqq \alpha_{n}(T) \leqq \min \left(\frac{\lambda_{n}}{2}, \lambda_{n+1}\right), \quad \frac{1}{r}+\frac{1}{q}=\frac{1}{p} .
$$

We mention that (4) above for $p \neq \infty$ has been computed by P. Johnson [3] and asymptotic estimates for $p=\infty$ have been given by Pietsch [8]. We are unable to compute $\alpha_{n}(T)$ exactly in case (5). However, there are operators $T, S: l_{1} \rightarrow l_{\infty}$ corresponding to decreasing sequences $\left(\lambda_{n}\right)$ and 
$\left(\beta_{n}\right)$ respectively such that $\alpha_{n}(T)=\left(\sum_{1}^{n+1} \lambda_{\imath}^{-1}\right)^{-1}$ and $\alpha_{n}(S)=\beta_{n} / 2$ for each $n$. Thus (5) gives the best asymptotic values of $\alpha_{n}$ possible.

For the remaining case, we remark that it is well known (see, e.g., [7]) that for $T: l_{p} \rightarrow l_{p}$ corresponding to a decreasing sequence $\left(\lambda_{n}\right), d_{n}(T)=$ $\alpha_{n}(T)=\lambda_{n+1}$ for each $n$.

3. $H$-operators and $\varepsilon_{p}$-finite bases. An operator $T$ acting on a Banach space $E$ is an $H$-operator if its spectrum is real and its resolvent $\rho(T)$ satisfies

$$
\left\|(T-\lambda I)^{-1}\right\| \leqq C\left|I_{m} \lambda\right|^{-1} \quad\left(I_{m} \lambda \neq 0\right), \quad C \text { independent of } \rho(T) .
$$

An operator on a Hilbert space is an $H$-operator with constant $C=1$ if and only if it is a selfadjoint operator. Markü [5] has proved that for a compact $H$-operator $T$ with eigenvalues $\left(\lambda_{n}\right)$ (numbered in order of decreasing modulus and taking into account their multiplicity), the sequences $\left(d_{n}(T)\right),\left(\alpha_{n}(T)\right)$ and $\left(\lambda_{n}\right)$ are equivalent, i.e., there are constants $A, B$ such that $d_{n}(T) \leqq \alpha_{n}(T) \leqq A\left|\lambda_{n+1}\right| \leqq B d_{n}(T)$.

We generalize this result to certain types of diagonal maps. We say that a (Schauder) basis $\left(x_{n}\right)$ for a Banach space $E$ is $\varepsilon_{p}$-finite provided $\varepsilon_{p}\left(x_{n}\right)=$ $\sup _{\|f\|=1}\left(\sum_{n=1}^{\infty}\left|f\left(x_{n}\right)\right|^{p}\right)^{1 / p}<+\infty$.

3.1. THEOREM. Let $T$ be a diagonal basis mapping $e_{n} \rightarrow \lambda_{n} u_{n}$ where $\left(e_{n}, f_{n}\right)$ and $\left(u_{n}, g_{n}\right)$ are $S$ chauder bases for $E$ and $F$ respectively and $\left(\lambda_{n}\right)$ is a monotonically decreasing null sequence, $\lambda_{n} \neq 0$. Suppose that $\varepsilon_{1}\left(g_{n} \otimes e_{n}\right)$ and $\varepsilon_{1}\left(f_{n} \otimes u_{n}\right)$ are finite. Then

$$
\left[\varepsilon_{1}\left(g_{n} \otimes e_{n}\right)\right]^{-1} \lambda_{n+1} \leqq d_{n}(T) \leqq \alpha_{n}(T) \leqq\left[\varepsilon_{1}\left(f_{n} \otimes u_{n}\right)\right] \lambda_{n+1} \cdot
$$

In any infinite dimensional Banach space there are basic sequences satisfying the hypotheses of 3.1.

Motivated by this result and a classical result of S. Bernstein, we say that two Banach spaces $E$ and $F$ form a Bernstein pair if for any positive monotonic null sequence $\left(b_{n}\right)$ there is a $T \in \mathscr{L}(E, F)$ such that $\left(\alpha_{n}(T)\right)$ is equivalent to $\left(b_{n}\right)$.

3.2. THEOREM. Let $E$ and $F$ be arbitrary infinite dimensional Banach spaces. Then there are infinite dimensional spaces $E_{0}$ and $F_{0}$ in $E$ and $F$ respectively such that $\left\langle E_{0}, F_{0}\right\rangle$ is a Bernstein pair. Moreover, the operator $T$ satisfying the definition for $\left\langle E_{0}, E_{0}\right\rangle$ can be chosen to be a compact $H$ operator.

3.3. Proposition. If $E=E_{1} \oplus E_{2}$ and $F=F_{1} \oplus F_{2}$ and $\left\langle E_{i}, F_{i}\right\rangle$ is a Bernstein pair for $i=1$ or 2 , then $\langle E, F\rangle$ is a Bernstein pair. 
As we have observed, in general, $\alpha_{n}(T) \neq \alpha_{n}\left(T^{*}\right)$. Thus the next result is somewhat surprising.

3.4. Theorem. Suppose $T \in \mathscr{F}(E, F)$. Then $\alpha_{n}(T)=\alpha_{n}\left(T^{*}\right)$ for each $n$.

Recall that an operator is of type $l_{p}[8]$ if $\left(\alpha_{n}(T)\right) \in l_{p}$.

3.5. Corollary. An operator $T$ is of type $l_{p}$ if and only if $T^{*}$ is of type $l_{p}$.

Another immediate corollary to 3.4 is the following fact concerning Bernstein pairs.

3.6. Corollary. If $\langle E, F\rangle$ is a Bernstein pair, so is $\left\langle F^{\prime}, E^{\prime}\right\rangle$.

Also from 3.4 we obtain for operators in $\mathscr{F}(E, F)$ the dual result of 1.2 .

3.7. Corollary. Let $i$ be an embedding of a Banach space $F$ into $l_{\infty}(\Gamma)$. Then if $T \in \mathscr{F}(E, F)$ we have $\alpha_{n}(i T)=d_{n}(T)$ for all $n$.

Perhaps all couplings of Banach spaces form Bernstein pairs. Indeed, all the classical Banach spaces form Bernstein pairs.

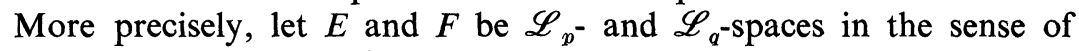
Lindenstrauss and Pełczyński [4]. Then $\langle E, F\rangle$ is a Bernstein pair.

Finally, we introduce the notion of a weak Bernstein pair. Two Banach spaces $E$ and $F$ form a weak Bernstein pair if there is a decreasing sequence $\left(b_{n}\right) \in c_{0} \backslash l_{2}$ and $T \in \mathscr{L}(E, F)$ such that $\left(\alpha_{n}(T)\right)$ is equivalent to $\left(b_{n}\right)$. Clearly a Bernstein pair is a weak Bernstein pair.

3.8. THEOREM. Any of the following is sufficient in order that Banach spaces $E$ and $F$ form a weak Bernstein pair.

(1) E contains an unconditional basic sequence;

(2) $E$ and $F$ have the approximation property;

(3) $E$ and $F$ admit equivalent uniformly convex norms.

The importance of the notion of a weak Bernstein pair is shown by the following corollary which partially answers an unsolved problem of Grothendieck and Rosenthal.

3.9. Corollary. If $(E, E)$ forms a weak Bernstein pair then on $E$ there is a compact nonnuclear operator.

ADDED IN REMARKS. Since submitting this abstract we have received a preprint from Professor A. Pietsch entitled s-numbers of bounded linear operators. In this paper, he develops an axiomatic theory of $s$-numbers: the $\alpha_{n}$ and $d_{n}$ above are special cases of this concept. Professor Pietsch proves many interesting results disjoint from our paper and also observes our Theorems 1.1 and 1.2 and the result of P. Johnson, 2.2(4). 


\section{REFERENCES}

1. I. C. Gohberg and M. G. Kreĭn, Introduction to the theory of linear nonselfadjoint operators in Hilbert space, "Nauka," Moscow, 1965; English transl., Transl. Math. Monographs, vol. 18, Amer. Math. Soc., Providence, R.I., 1969. MR 36 \#3137; 39 \#7447.

2. A. Grothendieck, Produits tensoriels topologiques et espaces nucléaires, Mem. Amer. Math. Soc. No. 16 (1955). MR 17, 763.

3. P. Johnson, Thesis, University of Michigan, Ann Arbor, Mich., 1973.

4. J. Lindenstrauss and A. Pelczyński, Absolutely summing operators in $\mathscr{L}_{p}$-spaces and their applications, Studia Math. 29 (1968), 275-326. MR 37 \#6743.

5. A. S. Markus, Some criteria for the completeness of a system of root vectors of a linear operator in a Banach space, Mat. Sb. 70 (112) (1966), 526-561; English transl., Amer. Math. Soc. Transl. (2) 85 (1969), 51-91. MR 35 \#7151.

6. A. S. Markus and V. I. Macaev, Analogs of Weyl inequalities and trace theorems in a Banach space, Mat. Sb. 86 (128) (1971), 299-313= Math. USSR Sb. 15 (1971), 299334. MR 45 \#7512.

7. B. S. Mitjagin and A. Pełczyński, Nuclear operators and approximative dimension, Proc. Internat. Congr. Math. (Moscow, 1966), "Mir," Mosców, 1968, pp. 366-372; English transl., Amer. Math. Soc. Transl. (2) 70 (1968), 137-145. MR 39 \#6046.

8. A. Pietsch, Einige neue Klassen von kompakten linearen Abbildungen, Rev. Math. Pures Appl. (Bucharest), 8 (1963), 427-447. MR 31 \#3874.

Department of Mathematics, Louisiana State University, Baton Rouge, LOUISIANA 70803 